\title{
On the connection between probability boxes and possibility measures
}

\author{
Matthias C. M. Troffaes ${ }^{1}$ Enrique Miranda $^{2}$ Sebastien Destercke $^{3}$ \\ ${ }^{1}$ Durham University, Durham, United Kingdom \\ ${ }^{2}$ University of Oviedo, Oviedo, Spain \\ ${ }^{3}$ UMR1208, French Agricultural Research Centre for International Development, Montpellier, France
}

\begin{abstract}
We explore the relationship between p-boxes on totally preordered spaces and possibility measures. We start by demonstrating that only those p-boxes who have $0-1$ valued lower or upper cumulative distribution function can be possibility measures, and we derive expressions for their natural extension in this case. Next, we establish necessary and sufficient conditions for a p-box to be a possibility measure. Finally, we show that almost every possibility measure can be modelled by a p-box. Whence, any techniques for p-boxes can be readily applied to possibility measures. We demonstrate this by deriving joint possibility measures from marginals, under varying assumptions of independence, using a technique known for $\mathrm{p}$-boxes.
\end{abstract}

Keywords: Possibility measures, maxitive measures, pboxes, coherent upper previsions, natural extension.

\section{Introduction}

Possibility measures [1] are supremum preserving set functions, and are widely applied in many fields, including data analysis [2], cased-based reasoning [3], and psychology [4]. In this paper we are concerned with quantitative possibility theory [5], where degrees of possibility range in the unit interval. Their interpretation as upper probability [6, 7] fits our purpose best.

Probability boxes [8], or p-boxes for short, are pairs of lower and upper cumulative distribution functions, and are often used in risk and safety studies, in which cumulative distributions play an essential role. P-boxes have been connected to info-gap theory [9], random sets [10], and also, partly, to possibility measures [11]. P-boxes can be defined on arbitrary finite spaces [12], and, more generally, even on arbitrary totally preordered spaces [13]—-we will use this extensively.

This paper aims to consolidate the connection between possibility measures and p-boxes, making as few assumptions as possible. We prove that almost every possibility measure can be interpreted as a p-box. Conversely, we provide necessary and sufficient conditions for a p-box to be a possibility measure.

To study this connection, we use imprecise probabilities [14], of which both possibility measures and pboxes are particular cases. Possibility measures are explored as imprecise probabilities in [6, 7, 15], and pboxes are studied as imprecise probabilities briefly in
[14. Section 4.6.6] and [16], and in much more detail in [13].

The paper is organised as follows: in Section 2, we give the basics of the behavioural theory of imprecise probabilities, and recall some facts about p-boxes and possibility measures; in Section 3, we first determine necessary and sufficient conditions for a p-box to be maximum preserving, before determining in Section 4 necessary and sufficient conditions for a p-box to be a possibility measure; in Section 5 , we show that almost any possibility measure can be seen as particular p-box, and that many p-boxes can be seen as a couple of possibility measures; some special cases are detailed in Section 6 Finally, in Section 7 we apply the work on multivariate p-boxes from [13] to derive multivariate possibility measures from given marginals, and in Section 8 we give a number of additional comments and remarks.

Note that proofs are omitted for brevity, whence beware that results are presented in the order that they appear most logical, and not in the order that they are most easily proven.

\section{Preliminaries}

\subsection{Imprecise Probabilities}

We briefly introduce imprecise probabilities; see [17, 18, 14, 19] for more details.

Let $\Omega$ be a possibility space. A subset of $\Omega$ is called an event. Denote the set of all events by $\wp(\Omega)$, and the set of all finitely additive probabilities on $\wp(\Omega)$ by $\mathscr{P}$.

An upper probability is any real-valued function $\bar{P}$ defined on an arbitrary subset $\mathscr{K}$ of $\wp(\Omega)$. With $\bar{P}$, we associate a lower probability $\underline{P}$ on $\left\{A: A^{c} \in \mathscr{K}\right\}$ via $\underline{P}(A)=1-\bar{P}\left(A^{c}\right)$. Consider the set

$$
\mathscr{M}(\bar{P})=\{P \in \mathscr{P}:(\forall A \in \mathscr{K})(P(A) \leq \bar{P}(A))\} .
$$

The upper envelope $\bar{E}$ of $\mathscr{M}(\bar{P})$ is called the natural extension [14, Thm. 3.4.1] of $\bar{P}$ :

$$
\bar{E}(A)=\sup \{P(A): P \in \mathscr{M}(\bar{P})\}
$$

for all $A \subseteq \Omega$. The corresponding lower probability is denoted by $\underline{E}$, so $\underline{E}(A)=1-\bar{E}\left(A^{c}\right)$. Clearly, $\underline{E}$ is the lower envelope of $\mathscr{M}(\bar{P})$.

We say that $\bar{P}$ is coherent (see [14, Sec. 3.3.3]) when, for all $A \in \mathscr{K}$,

$$
\bar{P}(A)=\bar{E}(A) .
$$

$\underline{P}$ is called coherent whenever $\bar{P}$ is. 


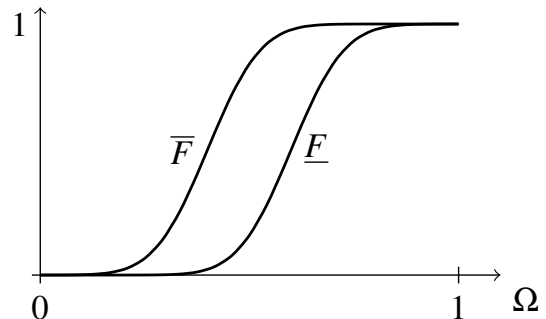

Figure 1: Example of a p-box on $[0,1]$.

\subsection{P-Boxes}

In this section, we revise p-boxes defined on totally preordered (not necessarily finite) spaces. For further details, see [13].

Start with a totally preordered space $(\Omega, \preceq)$. So, $\preceq$ is transitive, reflexive and any two elements are comparable. As usual, we write $x \prec y$ for $x \preceq y$ and $x \nsucceq y, x \succ y$ for $y \prec x$, and $x \simeq y$ for $x \preceq y$ and $y \preceq x$. For any two $x$, $y \in \Omega$ exactly one of $x \prec y, x \simeq y$, or $x \succ y$ holds. We also use the following common notation for intervals in $\Omega$ :

$$
\begin{aligned}
& {[x, y]=\{z \in \Omega: x \preceq z \preceq y\}} \\
& (x, y)=\{z \in \Omega: x \prec z \prec y\}
\end{aligned}
$$

and similarly for $[x, y)$ and $(x, y]$.

We assume that $\Omega$ has a smallest element $0_{\Omega}$ and a largest element $1_{\Omega}$-we can always add these two elements to the space $\Omega$.

A cumulative distribution function is a nondecreasing map $F: \Omega \rightarrow[0,1]$ for which $F\left(1_{\Omega}\right)=1$. For each $x \in \Omega, F(x)$ is interpreted as the probability of $\left[0_{\Omega}, x\right]$. $\Omega / \simeq$ :

The quotient set of $\Omega$ with respect to $\simeq$ is denoted by

$$
\begin{aligned}
{[x]_{\simeq} } & =\{y \in \Omega: y \simeq x\} \text { for any } x \in \Omega \\
\Omega / \simeq & =\left\{[x]_{\simeq}: x \in \Omega\right\} .
\end{aligned}
$$

Because $F$ is non-decreasing, $F$ is constant on elements $[x] \simeq$ of $\Omega / \simeq$.

Definition 1. A probability box, or $p$-box, is a pair $(\underline{F}, \bar{F})$ of cumulative distribution functions from $\Omega$ to $[0,1]$ satisfying $\underline{F} \leq \bar{F}$.

A p-box is interpreted as a lower and an upper cumulative distribution function (see Fig. 1), or more specifically, as an upper probability $\bar{P}_{F, \bar{F}}$ on the set of events

$$
\left\{\left[0_{\Omega}, x\right]: x \in \Omega\right\} \cup\left\{\left(y, 1_{\Omega}\right]: y \in \Omega\right\}
$$

defined by

$$
\bar{P}_{\underline{F}, \bar{F}}\left(\left[0_{\Omega}, x\right]\right)=\bar{F}(x) \text { and } \bar{P}_{\underline{F}, \bar{F}}\left(\left(y, 1_{\Omega}\right]\right)=1-\underline{F}(y) .
$$

We denote by $\bar{E}_{\underline{F}, \bar{F}}$ the natural extension of $\bar{P}_{\underline{F}, \bar{F}}$ to all events. To simplify the expression for natural extension, we introduce an element $0_{\Omega}-$ such that:

$$
\begin{gathered}
0_{\Omega}-\prec x \text { for all } x \in \Omega \\
F\left(0_{\Omega}-\right)=\underline{F}\left(0_{\Omega}-\right)=\bar{F}\left(0_{\Omega}-\right)=0 .
\end{gathered}
$$

Note that $\left(0_{\Omega}-, x\right]=\left[0_{\Omega}, x\right]$. Now, let $\Omega^{*}=\Omega \cup\left\{0_{\Omega}-\right\}$, and define

$$
\begin{aligned}
\mathscr{H}=\left\{\left(x_{0}, x_{1}\right] \cup\left(x_{2}, x_{3}\right]\right. & \cup \cdots \cup\left(x_{2 n}, x_{2 n+1}\right]: \\
x_{0} & \left.\prec x_{1} \prec \cdots \prec x_{2 n+1} \text { in } \Omega^{*}\right\} .
\end{aligned}
$$

Proposition 2. [13] Prop. 4] For any $A \in \mathscr{H}$, that is $A=$ $\left(x_{0}, x_{1}\right] \cup\left(x_{2}, x_{3}\right] \cup \cdots \cup\left(x_{2 n}, x_{2 n+1}\right]$ with $x_{0} \prec x_{1} \prec \cdots \prec$ $x_{2 n+1}$ in $\Omega^{*}$, it holds that $\bar{E}_{\underline{F}, \bar{F}}(A)=\bar{P}_{\underline{F}, \bar{F}}^{\mathscr{H}}(A)$, where

$$
\bar{P}_{\underline{F}, \bar{F}}^{\mathscr{H}}(A)=1-\sum_{k=0}^{n+1} \max \left\{0, \underline{F}\left(x_{2 k}\right)-\bar{F}\left(x_{2 k-1}\right)\right\},
$$

with $x_{-1}=0_{\Omega}-$ and $x_{2 n+2}=1_{\Omega}$.

To calculate $\bar{E}_{\underline{F}, \bar{F}}(A)$ for an arbitrary event $A \subseteq \Omega$, use the outer measure [14, Cor. 3.1.9] $\bar{P}_{\underline{F}, \bar{F}}^{\mathscr{H}}{ }^{*}$ of the upper probability $\bar{P}_{\underline{F}, \bar{F}}^{\mathscr{H}}$ defined in Eq. (1):

$$
\bar{E}_{\underline{F}, \bar{F}}(A)=\bar{P}_{\underline{F}, \bar{F}}^{\mathscr{H}}{ }^{*}(A)=\inf _{C \in \mathscr{H}, A \subseteq C} \bar{P}_{\underline{F}, \bar{F}}^{\mathscr{H}}(C) .
$$

For intervals, we immediately infer from Proposition 2 and Eq. (2) that ('i.p.' stands for 'immediate predecessor')

$$
\begin{aligned}
& \bar{E}_{\underline{F}, \bar{F}}((x, y])=\bar{F}(y)-\underline{F}(x) \\
& \bar{E}_{\underline{F}, \bar{F}}([x, y])=\bar{F}(y)-\underline{F}(x-) \\
& \bar{E}_{\underline{F}, \bar{F}}((x, y))= \begin{cases}\bar{F}(y)-\underline{F}(x) & \text { if } y \text { has no i.p. } \\
\bar{F}(y-)-\underline{F}(x) & \text { if } y \text { has an i.p. }\end{cases} \\
& \bar{E}_{\underline{F}, \bar{F}}([x, y))= \begin{cases}\bar{F}(y)-\underline{F}(x-) & \text { if } y \text { has no i.p. } \\
\bar{F}(y-)-\underline{F}(x-) & \text { if } y \text { has an i.p. }\end{cases}
\end{aligned}
$$

for any $x \prec y$ in $\Omega{ }^{1}$ where $\bar{F}(y-)$ denotes $\sup _{z \prec y} \bar{F}(z)$ and similarly for $\underline{F}(x-)$. If $\Omega / \simeq$ is finite, then one can think of $z$ - as the immediate predecessor of $z$ in the quotient space $\Omega / \simeq$ for any $z \in \Omega$. We also have that

$$
\bar{E}_{\underline{F}, \bar{F}}(\{x\})=\bar{F}(x)-\underline{F}(x-)
$$

for any $x \in \Omega$.

\subsection{Possibility and Maxitive Measures}

Brevity is stipulated; see [1, 5, 6, 7] for details.

Definition 3. A maxitive measure is an upper probability $\bar{P}$ on $\wp(\Omega)$ satisfying $\bar{P}(A \cup B)=\max \{\bar{P}(A), \bar{P}(B)\}$ for every $A$ and $B \subseteq \Omega$.

Proposition 4. [16] Def. 3.22, Thm. 3.46] A maxitive measure $\bar{P}$ is coherent whenever $\bar{P}(\emptyset)=0$ and $\bar{P}(\Omega)=1$.

Possibility measures are a particular case of maxitive measures.

\footnotetext{
${ }^{1}$ In case $x=0_{\Omega}$, evidently, $0_{\Omega}-$ is the immediate predecessor.
} 
Definition 5. A (normed) possibility distribution is a mapping $\pi: \Omega \rightarrow[0,1]$ satisfying $\sup _{x \in \Omega} \pi(x)=1$. A possibility distribution $\pi$ induces a possibility measure $\Pi$ on $\wp(\Omega)$, given by:

$$
\Pi(A)=\sup _{x \in A} \pi(x) \text { for all } A \subseteq \Omega .
$$

If we write $\underline{E}_{\Pi}$ for the conjugate lower probability of the upper probability $\Pi$, then:

$$
\underline{E}_{\Pi}(A)=1-\Pi\left(A^{c}\right)=1-\sup _{x \in A^{c}} \pi(x) .
$$

A possibility measure is maxitive, but not all maxitive measures are possibility measures.

As an imprecise probability model, possibility measures are not as expressive as for instance p-boxes. This poor expressive power is also illustrated by the fact that, for any event $A$ :

$$
\begin{aligned}
\Pi(A)<1 & \Longrightarrow \underline{E}_{\Pi}(A)=0, \quad \text { and } \\
\underline{E}_{\Pi}(A)>0 & \Longrightarrow \Pi(A)=1,
\end{aligned}
$$

meaning that every event has a trivial probability bound on at least one side. Their main attraction is that calculations with them are very easy: to find the upper (or lower) probability of any event, a simple supremum suffices.

In the following sections, we characterize the circumstances under which a possibility measure $\Pi$ is the natural extension of some p-box $(\underline{F}, \bar{F})$. In order to do so, we first characterise the conditions under which a p-box induces a maxitive measure.

\section{P-boxes as Maxitive Measures}

\subsection{Necessary and Sufficient Condition for Maxitivity}

Let us begin by characterising under which conditions the natural extension of a p-box is maxitive:

Theorem 6. The natural extension $\bar{E}_{\underline{F}, \bar{F}}$ of a p-box $(\underline{F}, \bar{F})$ is maximum preserving if and only if at least one of $\underline{F}$ or $\bar{F}$ is $0-1$-valued.

Hence, for the purposes of this paper we can restrict our attention to p-boxes $(\underline{F}, \bar{F})$ where at least one of $\underline{F}$ or $\bar{F}$ is $0-1$-valued. Next, we provide expressions for the natural extensions of such p-boxes, and determine under which conditions these are possibility measures.

\subsection{Natural Extension of Maxitive P-Boxes}

In case of $0-1$-valued $\underline{F}$, we arrive at the following expression:

Proposition 7. Let $(\underline{F}, \bar{F})$ be a p-box with 0 -1-valued $\underline{F}$, and let $B=\left\{x \in \Omega^{*}: \underline{F}(x)=0\right\}$. Then, for any $A \subseteq \Omega$,

$$
\bar{E}_{\underline{F}, \bar{F}}(A)=\min _{y \in B^{c}} \inf _{x \in \Omega^{*}:} \overline{A \cap\left[0_{\Omega}, y\right] \preceq x} \bar{F}(x) .
$$

Here, $A \preceq x$ means $z \preceq x$ for all $z \in A$, and similarly $y \prec A$ means $y \prec z$ for all $z \in A$. Thus, $\emptyset \preceq x$ and $y \prec \emptyset$ for all $x$ and $y$.

An important special case is summarized in the following corollary:

Corollary 8. Let $(\underline{F}, \bar{F})$ be a p-box with $0-1$-valued $\underline{F}$, and let $B=\left\{x \in \Omega^{*}: \underline{F}(x)=0\right\}$. If $\Omega / \simeq$ is order complete, then, for any $A \subseteq \Omega$,

$$
\bar{E}_{\underline{F}, \bar{F}}(A)=\min _{y \in B^{c}} \bar{F}\left(\sup A \cap\left[0_{\Omega}, y\right]\right) .
$$

If, in addition, $B^{c}$ has a minimum, then

$$
\bar{E}_{\underline{F}, \bar{F}}(A)=\bar{F}\left(\sup A \cap\left[0_{\Omega}, \min B^{c}\right]\right) .
$$

If, in addition, $\underline{F}=I_{\left[1_{\Omega}\right] \simeq \text {, then }}$

$$
\bar{E}_{\underline{F}, \bar{F}}(A)=\bar{F}(\sup A) \text {. }
$$

Note that Eq. (5) is essentially due to [7] paragraph preceeding Theorem 11] - they work with chains and multivalued mappings, whereas we work with total preorders. The case of order complete $\Omega / \simeq$ is applicable for instance when $\Omega$ is a finite space, or when it is an interval of real numbers.

In case of $0-1$-valued $\bar{F}$, we arrive at the following expression:

Proposition 9. Let $(\underline{F}, \bar{F})$ be a p-box with $0-1$-valued $\bar{F}$, and let $C=\left\{x \in \Omega^{*}: \bar{F}(x)=0\right\}$. Then, for any $A \subseteq \Omega$,

$$
\bar{E}_{\underline{F}, \bar{F}}(A)=1-\max _{x \in C} \sup _{y \in \Omega^{*}: y \prec A \cap\left(x, 1_{\Omega}\right]} \underline{F}(y) .
$$

Again, we summarize an important special case in the following corollary:

Corollary 10. Let $(\underline{F}, \bar{F})$ be a p-box with 0-1-valued $\bar{F}$, and let $C=\left\{x \in \Omega^{*}: \bar{F}(x)=0\right\}$. If $\Omega / \simeq$ is order complete, and $C$ has a maximum, then, for any $A \subseteq \Omega$,

$$
\bar{E}_{\underline{F}, \bar{F}}(A)= \begin{cases}1-\underline{F}\left(\inf A \cap C^{c}\right) & \text { if } A \cap C^{c} \text { has } \\ 1-\underline{F}\left(\left(\min A \cap C^{c}\right)-\right) & \text { no minimum } \\ & \text { if } A \cap C^{c} \text { has } \\ \text { a minimum }\end{cases}
$$

If, in addition, $\bar{F}=1$, then

$$
\bar{E}_{\underline{F}, \bar{F}}(A)= \begin{cases}1-\underline{F}(\inf A) & \text { if } A \text { has no minimum } \\ 1-\underline{F}(\min A-) & \text { if } A \text { has a minimum. }\end{cases}
$$

\section{P-Boxes as Possibility Measures.}

In this section, we identify when p-boxes coincide exactly with a possibility measure. All results in this section rely on the following trivial, yet important, lemma:

Lemma 11. For a p-box $(\underline{F}, \bar{F})$ there is a possibility measure $\Pi$ such that $\bar{E}_{\underline{F}, \bar{F}}=\Pi$ if and only if

$$
\bar{E}_{\underline{F}, \bar{F}}(A)=\sup _{x \in A} \bar{E}_{\underline{F}, \bar{F}}(\{x\}) \text { for all } A \subseteq \Omega
$$

and in such a case, $\pi(x)=\bar{E}_{\underline{F}, \bar{F}}(\{x\})$. 
We say that a p-box $(\underline{F}, \bar{F})$ is a possibility measure when the conditions of Lemma 11 are satisfied.

Taking into account that every possibility measure is maxitive, we deduce from Theorem 6 that, for a p-box to be a possibility measure, it is necessary that at least one of $\underline{F}$ or $\bar{F}$ is $0-1$-valued. The next corollary characterizes the additional regularity conditions to arrive at necessity and sufficiency:

Corollary 12. Assume that $\Omega / \simeq$ is order complete and let $(\underline{F}, \bar{F})$ be a p-box. Then $(\underline{F}, \bar{F})$ is a possibility measure if and only if either

(L1) $\underline{F}$ is 0-1-valued,

(L2) $\bar{F}(x)=\bar{F}(x-)$ for all $x \in \Omega$ that have no immediate predecessor, and

(L3) $\left\{x \in \Omega^{*}: \underline{F}(x)=1\right\}$ has a minimum,

or

(U1) $\bar{F}$ is 0-1-valued,

(U2) $\underline{F}(x)=\underline{F}(x+)$ for all $x \in \Omega$ that have no immediate successor, and

(U3) $\left\{x \in \Omega^{*}: \bar{F}(x)=0\right\}$ has a maximum.

Note that, in case $\underline{F}=I_{\left[1_{\Omega}\right]_{\simeq}}$, condition (L2) is essentially due to [7, Observation 9]. Also note that, for $\bar{E}_{F, \bar{F}}$ to be a possibility measure, the conditions are still necessary even when $\Omega / \simeq$ is not order complete: the proof in this direction does not require order completeness.

As a special case, when $\Omega / \simeq$ is finite, $\bar{E}_{F, \bar{F}}$ is a possibility measure with possibility distribution

$$
\pi(x)= \begin{cases}\bar{F}(x) & \text { if } x \preceq \min \{y: \underline{F}(y)=1\} \\ 0 & \text { otherwise. }\end{cases}
$$

when $\underline{F}$ is $0-1$-valued, and

$$
\pi(x)= \begin{cases}1-\underline{F}(x-) & \text { if } \bar{F}(x)=1 \\ 0 & \text { otherwise. }\end{cases}
$$

when $\bar{F}$ is $0-1$-valued.

We now characterise when, conversely, possibility measures can be represented as p-boxes. We shall see that this is possible for almost all of them.

\section{From Possibility Measures to P-Boxes}

\subsection{Possibility Measures as Specific P-Boxes}

[11] already discuss the link between possibility measures and p-boxes defined on the real line with the usual ordering. They show that any possibility measure can be approximated by a p-box, however at the expense of losing some information. We substantially strengthen their result, and even reverse it: we prove that any possibility measure with compact range can be exactly represented by a p-box with vacuous lower cumulative distribution function, that is, with $\underline{F}=I_{\left[1_{\Omega}\right]_{\simeq}}$. In other words, generally speaking, possibility measures are a special case of p-boxes on totally preordered spaces.
Theorem 13. For every possibility measure $\Pi$ on $\Omega$ with possibility distribution $\pi$ such that $\pi(\Omega)=\{\pi(x): x \in$ $\Omega\}$ is compact, there is a preorder $\preceq$ on $\Omega$ and an upper cumulative distribution function $\bar{F}$ such that the p-box $\left(\underline{F}=I_{\left[1_{\Omega}\right]_{\simeq}}, \bar{F}\right)$ is a possibility measure with possibility distribution $\pi$. In fact, one may take the preorder $\preceq$ to be the one induced by $\pi$ ( so $x \preceq y$ whenever $\pi(x) \leq \pi(y)$ ) and $\bar{F}=\pi$.

The representing $\mathrm{p}$-box is not necessarily unique:

Example 14. Let $\Omega=\left\{x_{1}, x_{2}\right\}$ and let $\Pi$ be the possibility measure determined by the possibility distribution

$$
\pi\left(x_{1}\right)=0.5 \quad \pi\left(x_{2}\right)=1 .
$$

By Theorem 13 , this possibility measure can be obtained if we consider the order $x_{1} \prec x_{2}$ and the p-box $\left(\underline{F}_{1}, \bar{F}_{1}\right)$ given by

$$
\begin{array}{ll}
\underline{F}_{1}\left(x_{1}\right)=0 & \underline{F}_{1}\left(x_{2}\right)=1 \\
\bar{F}_{1}\left(x_{1}\right)=0.5 & \bar{F}_{1}\left(x_{2}\right)=1 .
\end{array}
$$

However, we also obtain it if we consider the order $x_{2} \prec$ $x_{1}$ and the p-box $\left(\underline{F}_{2}, \bar{F}_{2}\right)$ given by

$$
\begin{array}{ll}
\underline{F}_{2}\left(x_{1}\right)=1 & \underline{F}_{2}\left(x_{2}\right)=0.5 \\
\bar{F}_{2}\left(x_{1}\right)=1 & \bar{F}_{2}\left(x_{2}\right)=1 .
\end{array}
$$

Indeed, by Corollary $12, \bar{E}_{\underline{F}_{2}, \bar{F}_{2}}$ is a possibility measure. By Eq. (4), its associated possibility distribution is

$$
\begin{aligned}
& \bar{E}_{\underline{F}_{2}, \bar{F}_{2}}\left(x_{2}\right)=\bar{F}\left(x_{2}\right)-\underline{F}\left(x_{2}-\right)=1 \\
& \bar{E}_{\underline{F}_{2}, \bar{F}_{2}}\left(x_{1}\right)=\bar{F}\left(x_{1}\right)-\underline{F}\left(x_{1}-\right)=0.5,
\end{aligned}
$$

i.e., $\pi$, as with the given ordering, $x_{2}-=0_{\Omega}-$ and $x_{1}-=x_{2}$.

Moreover, a p-box may be maximum preserving even if neither the lower nor the upper distribution function is vacuous, as we have shown in Corollary 12 .

Also, there are possibility measures which cannot be represented as p-boxes when $\pi(\Omega)$ is not compact:

Example 15. Let $\Omega=[0,1]$, and consider the possibility distribution given by $\pi(x)=(1+2 x) / 8$ if $x<0.5$, $\pi(0.5)=0.4$ and $\pi(x)=x$ if $x>0.5$; note that $\pi(\Omega)=$ $[0.125,0.25) \cup\{0.4\} \cup(0.5,1]$ is not compact. The ordering induced by $\pi$ is the usual ordering on $[0,1]$. Let $\Pi$ be the possibility measure induced by $\pi$. We show that there is no p-box $(\underline{F}, \bar{F})$ on $([0,1], \preceq)$, regardless of the ordering $\preceq$ on $[0,1]$, such that $\bar{E}_{\underline{F}, \bar{F}}=\Pi$.

By Corollary 12 , if $\bar{E}_{F, \bar{F}}=\Pi$, then at least one of $\underline{F}$ or $\bar{F}$ is $0-1-$ valued. Assume first that $F$ is $0-1-$ valued. By Eq. (4), $\bar{E}_{\underline{F}, \bar{F}}(\{x\})=\bar{F}(x)-\underline{F}(x-)=\pi(x)$. Because $\pi(x)>0$ for all $x$, it must be that $\underline{F}(x-)=0$ for all $x$, so $\bar{F}=\pi$. Because $\bar{F}$ is non-decreasing, $x \preceq y$ if and only if $\bar{F}(x) \leq \bar{F}(y)$; in other words, $\preceq$ can only be the usual ordering on $[0,1]$ for $(\underline{F}, \bar{F})$ to be a p-box. Hence, $\underline{F}=I_{\{1\}}$.

For $(\underline{F}, \bar{F})$ to induce the possibility measure $\Pi$, we know from Corollary 12 that $\bar{F}(x)=\bar{F}(x-)$ for every 
$x$ that has no immediate predecessor, that is, for every $x>0$. But, $\bar{F}(0.5)=0.4 \neq 0.25=\bar{F}(0.5-)$.

Similarly, if $\bar{F}$ would be $0-1$-valued, then we deduce from Eq. (4) that $\bar{F}(x)=1$ for every $x$, again because $\pi(x)>0$ for all $x$. Therefore, $F(x-)=1-\pi(x)$ for all $x$. But, because $\underline{F}$ is non-decreasing, one can easily show that $\preceq$ can only be the inverse of the usual ordering on $[0,1]$ for $(\underline{F}, \bar{F})$ to be a p-box.

Now, for $(\underline{F}, \bar{F})$ to induce the possibility measure $\Pi$, we know from Corollary 12 that $\underline{F}(x)=\underline{F}(x+)$ for every $x$ that has no immediate successor in with respect to $\preceq$, that is, for every $x \prec 0$, or equivalently, for every $x>0$. Whence,

$$
\underline{F}(x)=\underline{F}(x+)=\inf _{y \succ x} \underline{F}(y-)=1-\sup _{y<x} \pi(y)
$$

for all $x>0$. This leads to a contradiction: by the definition of $\pi$, we have on the one hand,

$$
\underline{F}(0.5-)=\sup _{x>0.5}\left(1-\sup _{y<x} \pi(y)\right)=0.5
$$

and on the other hand,

$$
\underline{F}(0.5-)=1-\pi(0.5)=0.6 .
$$

Hence, $\bar{E}_{F, \bar{F}}$ coincides with $\Pi$ in neither case.

Another way of relating possibility measures and pboxes goes via random sets (see for instance [10] and [12]).

\subsection{P-boxes as Conjunction of Possibility Measures}

In [12], where p-boxes are studied on finite spaces, it is shown that a p-box can be interpreted as the conjunction of two possibility measures, in the sense that $\mathscr{M}\left(\bar{P}_{F}, \bar{F}\right)$ is the intersection of two sets of additive probabilities induced by two possibility measures. The next proposition extends this result to arbitrary totally preordered spaces.

Proposition 16. Let $(\underline{F}, \bar{F})$ be a p-box such that $\left(\underline{F}, I_{\Omega}\right)$

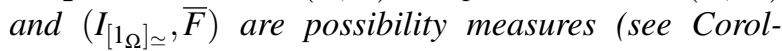
lary 12$)$. Then, $(\underline{F}, \bar{F})$ is the intersection of two possibility measures defined by the distributions

$$
\pi_{\bar{F}}(x)=\bar{F}(x) \text { and } \pi_{\underline{F}}(x)=1-\underline{F}(x-),
$$

in the sense that $\mathscr{M}\left(\bar{P}_{\underline{F}, \bar{F}}\right)=\mathscr{M}\left(\Pi_{\pi_{\bar{F}}}\right) \cap \mathscr{M}\left(\Pi_{\pi_{\underline{F}}}\right)$.

This suggests a simple way (already mentioned in [12]) to conservatively approximate $\underline{E}_{F}, \bar{F}$ by using the two possibility distributions: it holds that

$$
\begin{aligned}
\max \left\{\underline{E}_{\pi_{\bar{F}}}(A), \underline{E}_{\pi_{\underline{F}}}(A)\right\} \leq \underline{E}_{\underline{F}, \bar{F}}(A) \\
\\
\leq \bar{E}_{\underline{E}, \bar{F}}(A) \leq \min \left\{\bar{E}_{\pi_{\bar{F}}}(A), \bar{E}_{\pi_{\underline{F}}}(A)\right\} .
\end{aligned}
$$

This approximation is computationally attractive, as it allows us to use the supremum preserving properties of possibility measures. However, as next example shows, the approximation will usually be very conservative, and hence not likely to be helpful.
Example 17. Consider $x \prec y \in \Omega$. The distance between $\bar{E}_{\underline{F}, \bar{F}}$ and its approximation $\min \left\{\bar{E}_{\pi_{\bar{F}}}, \bar{E}_{\pi_{\underline{F}}}\right\}$ on the interval $(x, y]$ is given by

$$
\begin{aligned}
& \min \left\{\bar{E}_{\pi_{\bar{F}}}((x, y]), \bar{E}_{\pi_{F}}((x, y])\right\}-\bar{E}((x, y]) \\
& =\min \{\bar{F}(y), 1-\underline{F}(x)\}-(\bar{F}(y)-\underline{F}(x)) \\
& =\min \{\underline{F}(x), 1-\bar{F}(y)\} .
\end{aligned}
$$

Therefore, the approximation will be close to the exact value only when either $\underline{F}(x)$ is close to zero or $\bar{F}(y)$ is close to one.

In general, the set $\mathscr{M}\left(\Pi_{1}\right) \cap \mathscr{M}\left(\Pi_{2}\right)$ associated with two possibility distributions $\pi_{1}$ and $\pi_{2}$ is not a p-box. Indeed, it may be empty (take for instance $\Omega=\{1,2\}$ and the possibility measures determined by the distributions $\left.\pi_{1}(1)=1, \pi_{1}(2)=0.25, \pi_{2}(1)=0.25, \pi_{2}(2)=1\right)$, or result in another uncertainty model, such as a cloud [20]. In particular, when $\mathscr{M}\left(\Pi_{1}\right) \cap \mathscr{M}\left(\Pi_{2}\right)$ is non-empty, the resulting coherent upper probability is in general not a possibility measure. An interesting open problem is then to characterise under which conditions some of the properties (i.e., maxitivity, complete monotonicity) of $\Pi_{1}$ and $\Pi_{2}$ still hold for the coherent upper probability determined by $\mathscr{M}\left(\Pi_{1}\right) \cap \mathscr{M}\left(\Pi_{2}\right)$.

\section{Natural Extension of 0-1-Valued P-Boxes}

From Proposition 7, we can derive an expression for the natural extension of a $0-1$-valued p-box:

Proposition 18. Let $(\underline{F}, \bar{F})$ be a p-box where $\bar{F}=$ $I_{C^{c}}, \underline{F}=I_{B^{c}}$ for some $C \subseteq B \subseteq \Omega$. Then for any $A \subseteq \Omega$,

$\bar{E}_{\underline{F}, \bar{F}}(A)= \begin{cases}0 & \text { if there are } x \in C \text { and } y \in B^{c} \text { s.t. } \\ & A \cap C \preceq x, A \cap B \cap C^{c}=\emptyset, y \prec A \cap B^{c} \\ 1 \quad & \text { otherwise. }\end{cases}$

Moreover, Corollary 12 allows us to determine when this p-box is a possibility measure:

Proposition 19. Assume that $\Omega / \simeq$ is order complete. Let $(\underline{F}, \bar{F})$ be a p-box where $\bar{F}=I_{C^{c}}, \underline{F}=I_{B^{c}}$ for some $C \subseteq B \subseteq \Omega$. The following statements are equivalent:

\section{1. $(\underline{F}, \bar{F})$ is a possibility measure.}

\section{2. $B^{c}$ has a minimum and $C$ has a maximum.}

In particular, we can characterise under which conditions a precise p-box, i.e., one where $\underline{F}=\bar{F}:=F$, induces a possibility measure. The natural extension of precise p-boxes on the unit interval was considered in [21. Section 3.1]. From Theorem 6, the natural extension of $F$ can only be a possibility measure when $F$ is $0-1$-valued. If we apply Propositions 18 and 19 with $B=C$ we obtain the following:

Corollary 20. Let $(\underline{F}, \bar{F})$ be a precise p-box where $\bar{F}=$ $\underline{F}$ is $0-1$-valued, and let $B=\left\{x \in \Omega^{*}: \underline{F}(x)=0\right\}$. Then, for every subset $A$ of $\Omega$,

$$
\bar{E}_{\underline{F}, \bar{F}}(A)= \begin{cases}0 & \text { if there are } x \in B, y \in B^{c} \\ & \text { s.t. } A \cap B \preceq x \text { and } y \prec A \cap B^{c} \\ 1 & \text { otherwise. }\end{cases}
$$


If moreover $\Omega / \simeq$ is order complete, then $(\underline{F}, \bar{F})$ is a possibility measure if and only if $B$ has a maximum and $B^{c}$ has a minimum.

As a consequence, we deduce that a precise $0-1$ valued p-box never induces a possibility measure in case $(\Omega, \preceq)=([0,1], \leq)$, except if $F=I_{[0,1]}$.

\section{Constructing Multivariate Possibility Measures from Marginals}

In [13], multivariate p-boxes were constructed from marginals. We next apply this construction together with the $\mathrm{p}$-box representation of possibility measures, given by Theorem 13 to build a joint possibility measure from some given marginals. As particular cases, we consider the joint,

(i) either without any assumptions about dependence or independence between variables, that is, using the Fréchet-Hoeffding bounds [22],

(ii) or assuming epistemic independence between all variables, which allows us to use the factorization property [23].

Let us consider $n$ variables $X_{1}, \ldots, X_{n}$, and assume that for each variable $X_{i}$ we are given a possibility measure $\Pi_{i}$ with corresponding possibility distribution $\pi_{i}$ on $\mathscr{X}_{i}$. We assume that the range of all marginal possibility distributions is $[0,1]$; in particular, Theorem 13 applies, and each marginal can be represented by a p-box on $\left(\mathscr{X}_{i}, \preceq_{i}\right)$, with vacuous $\underline{F}_{i}$, and $\bar{F}_{i}=\pi_{i}$. Remember that the preorder $\preceq_{i}$ is the one induced by $\pi_{i}$.

\subsection{Multivariate Possibility Measures}

The construction in [13, Sect. 7] employs the following mapping $Z$, which induces a preorder $\preceq$ on $\Omega=\mathscr{X}_{1} \times$ $\cdots \times \mathscr{X}_{n}$ :

$$
Z\left(x_{1}, \ldots, x_{n}\right)=\max _{i=1}^{n} \pi_{i}\left(x_{i}\right) .
$$

With this choice of $Z$, we can easily find the possibility measure which represents the joint as accurately as possible, under any rule of combination of coherent lower probabilities:

Lemma 21. Let $\odot$ be any rule of combination of coherent upper probabilities, mapping the marginals $\bar{P}_{1}, \ldots$, $\bar{P}_{n}$ to a joint coherent upper probability $\bigodot_{i=1}^{n} \bar{P}_{i}$ on all events. If there is a continuous function $u$ for which

$$
\bigodot_{i=1}^{n} \bar{P}_{i}\left(\prod_{i=1}^{n} A_{i}\right)=u\left(\bar{P}_{1}\left(A_{1}\right), \ldots, \bar{P}_{n}\left(A_{n}\right)\right)
$$

for all $A_{1} \subseteq \mathscr{X}_{1}, \ldots, A_{n} \subseteq \mathscr{X}_{n}$, then the possibility distribution $\pi$ defined by

$$
\pi(x)=u(Z(x), \ldots, Z(x))
$$

induces the least conservative upper cumulative distribution function on $(\Omega, \preceq)$ that dominates the combination $\bigodot_{i=1}^{n} \Pi_{i}$ of $\Pi_{1}, \ldots, \Pi_{n}$.
This result is a consequence of [13, Lemma 22], which gives the least conservative p-box that dominates the natural extension of the combination of some marginal p-boxes by $\odot$. Here we are considering the particular case where the marginal p-boxes represent possibility measures, and approximating of the corresponding p-box by a possibility measure.

\subsection{Natural Extension: The Fréchet Case}

The natural extension $\bigotimes_{i=1}^{n} \bar{P}_{i}$ of $\bar{P}_{1}, \ldots, \bar{P}_{n}$ is the upper envelope of all joint (finitely additive) probability measures whose marginal distributions are compatible with the given marginal upper probabilities. So, the model is completely vacuous (that is, it makes no assumptions) about the dependence structure, as it includes all possible forms of dependence. See [24, p. 120, \$3.1] for a rigorous definition. In this paper, we only need the following equality, which is one of the Fréchet bounds (see for instance [14, p. 122, §3.1.1]):

$$
\bigotimes_{i=1}^{n} \bar{P}_{i}\left(\prod_{i=1}^{n} A_{i}\right)=\min _{i=1}^{n} \bar{P}_{i}\left(A_{i}\right)
$$

for all $A_{1} \subseteq \mathscr{X}_{1}, \ldots, A_{n} \subseteq \mathscr{X}_{n}$.

Theorem 22. The possibility distribution

$$
\pi(x)=\max _{i=1}^{n} \pi_{i}\left(x_{i}\right)
$$

induces the least conservative upper cumulative distribution function on $(\Omega, \preceq)$ that dominates the natural extension $\otimes_{i=1}^{n} \Pi_{i}$ of $\Pi_{1}, \ldots, \Pi_{n}$.

In other words, if we consider the marginal credal sets $\mathscr{M}\left(\Pi_{1}\right), \ldots, \mathscr{M}\left(\Pi_{n}\right)$ and consider the set $\mathscr{M}$ of all the finitely additive probabilities on $\Omega$ whose $\mathscr{X}_{i}$-marginals belong to $\mathscr{M}\left(\Pi_{i}\right)$ for $i=1, \ldots, n$, then $\mathscr{M}$ is included in the credal set of the possibility distribution $\pi$ as defined in Eq. (6).

Since it is based on very mild assumptions, it is not surprising that the possibility distribution given by Eq. (6) is very uninformative (that is, very close to a vacuous model where $\pi(x)=1$ for every $x$ ): we shall have $\pi(x)=1$ as soon as $\pi_{i}\left(x_{i}\right)=1$ for some $i$, even if $\pi_{j}\left(x_{j}\right)=0$ for every $j \neq i$. In particular, if one of the marginal possibility distributions is vacuous, then so is $\pi$. This also shows that the corresponding possibility measure $\Pi$ may not have $\Pi_{1}, \ldots, \Pi_{n}$ as its marginals.

\subsection{Independent Natural Extension}

In contrast, we can consider joint models which satisfy the property of epistemic independence between the different $X_{1}, \ldots, X_{n}$. This property means, roughly speaking, that the conditional models that we can derive from the joint coincide with the marginals. The most conservative of these models is called the independent natural extension $\otimes_{i=1}^{n} \bar{P}_{i}$ of $\bar{P}_{1}, \ldots, \bar{P}_{n}$. See [23] for a rigorous definition and properties, and [15] for a study of joint possibility measures that satisfy epistemic independence in the case of two variables. In this paper, we only need 
the following equality for the independent natural extension:

$$
\bigotimes_{i=1}^{n} \bar{P}_{i}\left(\prod_{i=1}^{n} A_{i}\right)=\prod_{i=1}^{n} \bar{P}_{i}\left(A_{i}\right)
$$

for all $A_{1} \subseteq \mathscr{X}_{1}, \ldots, A_{n} \subseteq \mathscr{X}_{n}$.

Theorem 23. The possibility distribution

$$
\pi(x)=\left(\max _{i=1}^{n} \pi_{i}\left(x_{i}\right)\right)^{n}
$$

induces the least conservative upper cumulative distribution function on $(\Omega, \preceq)$ that dominates the independent natural extension $\otimes_{i=1}^{n} \Pi_{i}$ of $\Pi_{1}, \ldots, \Pi_{n}$.

Note that the possibility measure determined Eq. (8) is only an outer approximation of the independent natural extension: as shown in [15, Sec. 6], there is no least conservative possibility measure that corresponds to the independent natural extension of possibility measures.

One interesting consequence of Theorem 23 is that we can also use the possibility distribution determined by Eq. (8) under some other independence conditions for imprecise probabilities, such as strong or Kuznetsov independence [25, 26]. This is because the joint model they determine also satisfies the factorization condition given by Eq. (7), and as a consequence the least conservative upper cumulative distribution that dominates the strong or the Kuznetsov product of the marginal possibility measures is also determined by Eq. (8). See [23] for more information on the relationship between factorization and independence.

We do not consider the minimum rule and the product rule

$$
\min _{i=1}^{n} \pi_{i}\left(x_{i}\right) \text { and } \prod_{i=1}^{n} \pi_{i}\left(x_{i}\right)
$$

as their relation with the theory of coherent lower previsions is still unclear. However, we can compare the above approximation with the following outer approximation given by [27, Proposition 1]:

$$
\pi(x)=\min _{i=1}^{n}\left(1-\left(1-\pi_{i}\left(x_{i}\right)\right)^{n}\right) .
$$

The above equation is an outer approximation in case of random set independence, which is slightly more conservative than the independent natural extension [28, Sec. 4], so in particular, it is also an outer approximation of the independent natural extension. Essentially, each distribution $\pi_{i}$ is transformed into $1-\left(1-\pi_{i}\right)^{n}$ before applying the minimum rule. It can be expressed more simply as

$$
1-\max _{i=1}^{n}\left(1-\pi_{i}\left(x_{i}\right)\right)^{n}
$$

If for instance $\pi\left(x_{i}\right)=1$ for at least one $i$, then this formula provides a more informative (i.e., lower) upper bound than Theorem 23 . On the other hand, when all $\pi\left(x_{i}\right)$ are, say, less than $1 / 2$, then Theorem 23 does better.

Finally, note that neither Eq. (8) nor Eq. 9) are proper joints, in the sense that, in both cases, the marginals of the joint are outer approximations of the original marginals, and will in general not coincide with the original marginals.

\section{Conclusions}

Both possibility measures and p-boxes can be seen as coherent upper probabilities. We used this framework to study the relationship between possibility measures and p-boxes. Following [13], we allowed p-boxes to be defined on arbitrary totally preordered spaces, whence including p-boxes on finite spaces, on real intervals, and even multivariate ones.

We began by considering the more general case of maxitive measures, and proved that a necessary and sufficient condition for a p-box to be maxitive is that at least one of the cumulative distribution functions of the p-box must be $0-1$ valued. Moreover, we determined the natural extension of a p-box in those cases and gave a necessary and sufficient condition for the p-box to be supremum-preserving, i.e., a possibility measure. As special cases, we also studied p-boxes where both the lower and the upper distribution functions are 0-1valued, and in particular precise $0-1$ valued p-boxes.

Secondly, we showed that almost every possibility measure can be represented as a p-box. Hence, in general, p-boxes are more expressive than possibility measures, while still keeping a relatively simple representation and calculus [13], unlike many other models, such as for instance lower previsions and credal sets, which typically require far more advanced techniques, such as linear programming.

Finally, we considered the multivariate case in more detail, by deriving a joint possibility measure from given marginals using the p-box representation established in this paper and results from [13].

In conclusion, we established new connections between both models, strengthening known results from literature, and allowing many results from possibility theory to be embedded into the theory of p-boxes, and vice versa.

As future lines of research, we point out the generalisation of a number of properties of possibility measures to p-boxes, such as the connection with fuzzy logic [1] or the representation by means of graphical structures [29], and the study of the connection of p-boxes with other uncertainty models, such as clouds and random sets.

\section{Acknowledgements}

Work partially supported by projects TIN2008-06796C04-01 and MTM2010-17844 and by a doctoral grant from the IRSN.

\section{References}

[1] L. A. Zadeh. Fuzzy sets as a basis for a theory of possibility. Fuzzy Sets and Systems, 1:3-28, 1978. 
[2] H. Tanaka and P.J. Guo. Possibilistic Data Analysis for Operations Research. Physica-Verlag, Heidelberg, 1999.

[3] E. Hüllermeier. Cased-based approximate reasoning. Springer, 2007.

[4] E. Raufaste, R. Da Silva Neves, and C. Mariné. Testing the descriptive validity of possibility theory in human judgements of uncertainty. Artificial Intelligence, 148:197-218, 2003.

[5] D. Dubois and H. Prade. Possibility Theory. Plenum Press, New York, 1988.

[6] P. Walley. Measures of uncertainty in expert systems. Artificial Intelligence, 83:1-58, 1996.

[7] G. de Cooman and D. Aeyels. Supremum preserving upper probabilities. Information Sciences, 118:173-212, 1999.

[8] S. Ferson and W. Tucker. Sensitivity analysis using probability bounding. Reliability engineering and system safety, 91(10-11):1435-1442, 2006.

[9] S. Ferson and W. Tucker. Probability boxes as infogap models. In Proceedings of the Annual Meeting of the North American Fuzzy Information Processing Society, New York (USA), 2008.

[10] E. Kriegler and H. Held. Utilizing belief functions for the estimation of future climate change. International Journal of Approximate Reasoning, 39:185-209, 2005.

[11] C. Baudrit and D. Dubois. Practical representations of incomplete probabilistic knowledge. Computational Statistics and Data Analysis, 51(1):86108, 2006.

[12] S. Destercke, D. Dubois, and E. Chojnacki. Unifying practical uncertainty representations: I. Generalized p-boxes. International Journal of Approximate Reasoning, 49(3):649-663, 2008.

[13] M. C. M. Troffaes and S. Destercke. Probability boxes on totally preordered spaces for multivariate modelling. International Journal of Approximate Reasoning, 2011. In press.

[14] P. Walley. Statistical Reasoning with Imprecise Probabilities. Chapman and Hall, London, 1991.

[15] E. Miranda and G. de Cooman. Epistemic independence in numerical possibility theory. International Journal of Approximate Reasoning, 32:2342, 2003.

[16] M. C. M. Troffaes. Optimality, Uncertainty, and Dynamic Programming with Lower Previsions. $\mathrm{PhD}$ thesis, Ghent University, Ghent, Belgium, March 2005.

[17] G. Boole. An investigation of the laws of thought on which are founded the mathematical theories of logic and probabilities. Walton and Maberly, London, 1854.

[18] P. M. Williams. Notes on conditional previsions. Technical report, School of Mathematical and Physical Science, University of Sussex, UK, 1975.

[19] E. Miranda. A survey of the theory of coherent lower previsions. International Journal of Approximate Reasoning, 48(2):628-658, 2008.
[20] Didier Dubois and Henri Prade. Interval-valued fuzzy sets, possibility theory and imprecise probability. In In Proceedings of International Conference in Fuzzy Logic and Technology, pages 314$319,2005$.

[21] E. Miranda, G. de Cooman, and E. Quaeghebeur. Finitely additive extensions of distribution functions and moment sequences: the coherent lower prevision approach. International Journal of Approximate Reasoning, 48(1):132-155, 2008.

[22] W. Hoeffding. Probability inequalities for sums of bounded random variables. Journal of the Americal Statistical Association, 58:13-30, 1963.

[23] G. de Cooman, E. Miranda, and M. Zaffalon. Independent natural extension. In Eyke Hüllermeier, Rudolf Kruse, and Frank Hoffmann, editors, Computational Intelligence for Knowledge-Based Systems Design, Lecture Notes in Computer Science, pages 737-746. Springer, 2010.

[24] G. de Cooman and M. C. M. Troffaes. Coherent lower previsions in systems modelling: products and aggregation rules. Reliability Engineering and System Safety, 85:113-134, 2004.

[25] F. G. Cozman. Constructing sets of probability measures through Kuznetsov's independence condition. In G. de Cooman, T. L. Fine, and T. Seidenfeld, editors, ISIPTA 'O1 - Proceedings of the Second International Symposium on Imprecise Probabilities and Their Applications, pages 104-111. Shaker Publishing, Maastricht, 2000.

[26] G. de Cooman, E. Miranda, and M. Zaffalon. Factorisation properties of the strong product. In Christian Borgelt, Gil González-Rodríguez, Wolfang Trutschnig, Asunción Lubiano, Ángeles Gil, Przemyslaw Grzegorzewski, and Olgierd Hryniewciz, editors, Combining soft computing and statistical methods in data analysis, Advances in intelligent and soft computing, pages 139-148. Springer, 2010.

[27] S. Destercke, D. Dubois, and E. Chojnacki. Consonant approximation of the product of independent consonant random sets. International Journal of Uncertainty, Fuzziness and Knowledge-Based Systems, 17(6):773-792, 2009.

[28] I. Couso, S. Moral, and P. Walley. Examples of independence for imprecise probabilities. Risk Decision and Policy, 5:165-181, 2000.

[29] C. Borgelt, J. Gebhardt, and R. Kruse. Possibilistic graphical models. In G. Della Riccia, R. Kruse, and H.J. Lenz, editors, Computational Intelligence in Data Mining, pages 51-68. Springer, Berlin, 2000. 